
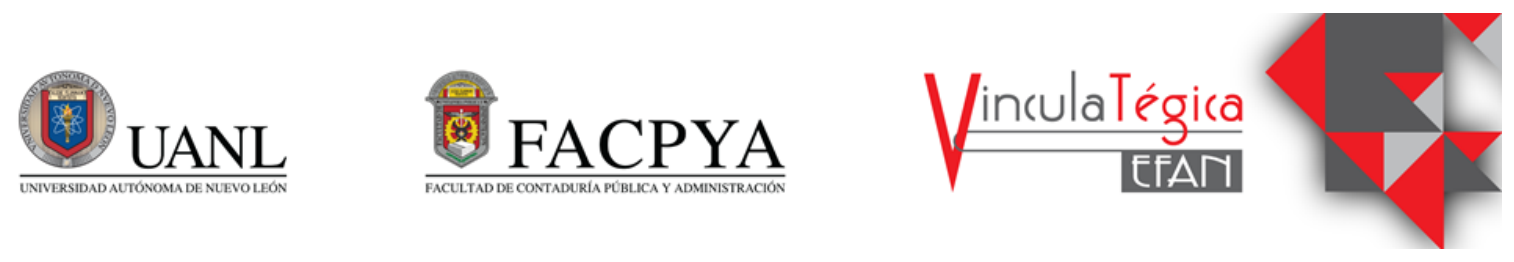

\title{
Evaluación en la calidad en servicios educativos universitarios: evidencia de estudiantes de economía en la pandemia de Covid-19
}

\author{
Jorge O. Moreno Treviño ${ }^{1}$; Alan García Gallegos ${ }^{2}$ \\ y Jacqueline Hernández Martínez ${ }^{3}$ \\ ${ }^{1}$ Universidad Autónoma de Nuevo León, Facultad de Economía, Monterrey, \\ N.L., jorge.morenotr@uanl.edu.mx, Ave. Lázaro Cárdenas 4600, UANL Campus Mederos, C.P. 64930, (+52) \\ 818-329-4150 (Ext.5402) \\ ${ }^{2}$ Universidad Autónoma de Nuevo León, Facultad de Economía, Monterrey, \\ N.L., alan.garciaga@uanl.edu.mx, Ave. Lázaro Cárdenas 4600, UANL Campus Mederos, C.P. 64930, (+52) \\ 818-329-4150 (Ext.5402) \\ ${ }^{3}$ Universidad Autónoma de Nuevo León, Facultad de Economía, Monterrey, \\ N.L., jacqueline.hernandezma@uanl.edu.mx, Ave. Lázaro Cárdenas 4600, UANL Campus Mederos, C.P. \\ 64930, (+52) 818-329-4150 (Ext.5402)
}

Información del artículo revisado por pares

Fecha de aceptación: junio-2021

Fecha de publicación en línea: octubre-2021

DOI: https://doi.org/10.29105/vtga7.2-3

\begin{abstract}
Resumen
Objetivo: analizar las dimensiones de calidad en los servicios educativos universitarios de la Facultad de Economía de la UANL desde la perspectiva de los estudiantes usuarios en el contexto del confinamiento por la pandemia de Covid-19. Método: se realizó un muestreo aleatorio estratificado para obtener representatividad en los ítems relevantes por sexo y para cada una de las generaciones. Se aplicó el instrumento SERVQUAL (Parasuraman, 1988) para medir "expectativas" y "percepción recibida" de los servicios educativos a una muestra representativa de 350 estudiantes, 142 mujeres y 208 hombres. Todos los items y dimensiones muestran validez en su aplicación de acuerdo con el criterio de Alfa de Cronbach. Posteriormente se utilizan pruebas de Satterwhite entre distintos grupos y estratos. Resultados: se encontró que las dimensiones en las que los alumnos tienen mayores expectativas son "seguridad" $y$ "empatía" mientras que las dimensiones con mejores niveles de percepción del servicio recibido son "tangibilidad" y "seguridad". La calidad global percibida implica un valor de -.763 , lo cual sugiere que la calidad es insatisfactoria en términos de los
\end{abstract}

\begin{abstract}
Objective: analyze the quality dimensions in the university educational services at Facultad de Economía of UANL, from the perspective of student users, in the context of confinement due to the Covid19 pandemic. Method: a stratified random sampling was carried out to obtain representativeness in the relevant items by sex and for each generation. The SERVQUAL instrument (Parasuraman, 1988) was applied to measure "expectations" and "received perception" of educational services to a representative sample of 350 students, 142 women and 208 men. All items and dimensions show validity in their application according to Cronbach's Alpha criterion. Subsequently, various Satterwhite tests are used for average levels and differences in means between different groups and strata. Results: it is found that the dimensions in which students have the highest expectations are "security" and "empathy," while the dimensions with the best levels of perception of the service received are "tangibility" and "security." The overall perceived quality implies a value of -.763 , which suggests that the faculty's quality is unsatisfactory in terms of the services received. Limitations: it is not possible to carry out
\end{abstract}


servicios recibidos. Limitaciones: no es posible realizar el mismo estudio para antes de la pandemia por lo que los resultados son condicionales al confinamiento.

Palabras clave: calidad de servicios educativos, expectativas, estudiantes universitarios, economistas, Covid-19.

\section{INTRODUCCIÓN}

La UNICEF menciona que "una educación de calidad, esencial para el aprendizaje verdadero y el desarrollo humano se ve influida por factores que proceden del interior y el exterior del aula como la existencia de unos suministros adecuados o la naturaleza del entorno doméstico del niño o niña. Además de facilitar la transmisión de conocimientos y aptitudes necesarias para triunfar en una profesión y romper el ciclo de pobreza, la calidad desempeña un papel crítico a la hora de disminuir la brecha existente entre los géneros en materia de educación básica". Es indispensable conocer cuáles son las necesidades de los estudiantes ya que ellos son los que se les está ofreciendo el servicio de la educación. Diseñar un proceso para mejorar la calidad de una institución sin tomar en cuenta la percepción estudiantil no tendría sentido ya que ellos son los principales consumidores de ese servicio.

Los estudiantes son una de las partes interesadas más importantes de la calidad educativa. Satisfacer a los estudiantes es uno de los propósitos principales en el sector educativo. Los estudiantes satisfechos son una fuente de ventaja competitiva y una fuente de marketing de boca a boca para las instituciones educativas. La satisfacción y la motivación de los estudiantes en el sector educativo pueden atribuirse a la provisión de calidad en el instituto. Para ver qué calidad satisface o motiva a los estudiantes se debe medir la calidad del instituto (Ahmed, 2010). La educación superior es un servicio ya que exhibe todas las características clásicas de los servicios: es intangible y heterogéneo, cumple el criterio de inseparabilidad al ser producido y consumido al mismo tiempo, satisface el the same study before the pandemic, so the results are conditional on confinement.

Keywords: educational services quality, expectations, university students, economists, Covid19.

criterio de perecibilidad y asume la participación en el proceso de entrega (Cuthbert, 1996). Los conceptos de calidad del servicio son directamente aplicables a la educación superior. La calidad de los servicios prestados por las instituciones de educación superior debe mejorarse continuamente para mantenerse al día con las demandas e intereses de sus partes interesadas (Đonlagić, 2015).

\section{REVISIÓN DE LITERATURA}

La calidad del servicio es ampliamente utilizada y experimentada en diversos sectores a través del modelo SERVQUAL (Parasuraman, 1988). Cinco dimensiones de la calidad del servicio son las dimensiones que se pueden aplicar en cada sector, ya sea el sector de fabricación o de servicios, y son: a) tangibilidad: instalaciones físicas, equipos y el aspecto del personal, b) fiabilidad: habilidad para realizar el servicio prometido (en este caso la educación) en forma fiable y precisa, c) capacidad de respuesta: disposición para ayudar a los clientes (estudiantes) y proporcionar un rápido servicio, d) seguridad: el conocimiento y la cortesía de los maestros y personal académico para inspirar confianza y seguridad y e) empatía: el cuidado y la atención individualizada que la institución proporciona a sus estudiantes.

Diversos estudios analizan la calidad de una universidad a través de la escala SERVQUAL midiendo la calidad percibida global por los estudiantes y las diferencias entre las percepciones y expectativas para cada una de las dimensiones del instrumento. Algunos autores que analizan la calidad en facultades de negocios y economía (Chua, 2004; Foropon et al., 2013; Pariseau, 1997; Djolagic, 2015; Goran, 2014; Barnes, 2007; Bautista et al., 2017) encuentran resultados parecidos en cuanto a la evaluación de las 
percepciones y expectativas de los estudiantes hacia su facultad. Cinco de los autores encuentran que la percepción de la empatía es de las más bajas evaluadas y la percepción de seguridad fue en promedio la mejor evaluada por parte de los estudiantes.

En cuanto a las expectativas la tangibilidad es la que en promedio fue evaluada más bajo. Todas las facultades presentan una calidad percibida negativa (insatisfactoria). Aunque Guzmán (2018) menciona que los resultados no indican que al ser negativas se tiene un mal servicio. Lo que da a entender y demuestra el SERVQUAL es que la calidad del servicio no sólo está enfocada en la percepción del servicio recibido sino también en las expectativas que tienen los usuarios sobre un servicio antes de que este sea brindado.

El análisis de la calidad en universidades a través de SERVQUAL es utilizado alrededor del mundo: Grecia (Ropoulos y Vrana, 2007), Escocia (Ibrahim et al., 2013), Estados Unidos (Foropon et al., 2013), México (Jaquez, 2017), Italia (Wael, 2015), India (Sahney et al., 2004), Irán (Aghamolaei et al., 2008), Serbia (Krsmanovic et al., 2014), Malasia (Chui et al., 2016) y Sudáfrica (Kanakana, 2014), entre otros. Los estudios revelan que en promedio las universidades que se encuentran en países más desarrollos presentan niveles de calidad percibida mejores que en países menos desarrollados. Las percepciones de las dimensiones por parte de los estudiantes son muy bajas en países menos desarrollados.

Además, en países de África y Asia como en Malasia (Shekarchizadeh et al., 2011; Yan Li et al., 2018; Rasli et al., 2012), Ghana (Banahene et al., 2017), India (Gopalakrishnan, 2014), Irán (Akhlaghi et al., 2012; Abili et al., 2012), Emiratos Árabes Unidos (Datta y Vardhan et al., 2017), Arabia Saudita (Alhabeeb, 2015) y Tailandia (Yousapronpaiboon, 2014) se hacen análisis entre las percepciones y expectativas para saber cuáles dimensiones de la escala SERVQUAL son evaluadas muy insatisfactoriamente para así dirigir mejor sus finanzas o planes de estudio para mejorar los aspectos que tienen un impacto en las percepciones de los estudiantes sobre la calidad del servicio que reciben.
También Akhlaghi (2012) menciona que la obtención de nuevos estudiantes y la retención de los estudiantes actuales siempre se ha considerado como principal motivo de su universidad, por esta razón utilizan la escala SERVQUAL para revelar las prioridades de modificación y mejora de los servicios de educación que dan. Los resultados muestran que las principales dimensiones donde deberían enfocarse son la capacidad de respuesta y la tangibilidad.

\section{ESTRATEGIA ESTADÍSTICA}

Los datos que se presentan en este trabajo de investigación proceden de una encuesta elaborada con un conjunto de instrumentos que permiten conocer: las habilidades cognitivas del estudiante, aptitudes académicas vocacionales, contexto socioeconómico, familiar y del hogar, hábitos de consumo, así como métricas de autoconcepto, perfeccionismo e inteligencias múltiples. En particular se utiliza el instrumento SERVQUAL adoptado a medir la calidad ofrecida a los estudiantes por parte de su facultad (Parasuraman, 1988). Este instrumento tiene como objetivo central medir las expectativas y percepciones de calidad ofrecidas y la diferencia entre éstas donde si las expectativas son mayores se tiene una calidad de servicio ofrecido insatisfactoria. El modelo SERVQUAL está compuesto de 50 items, 25 que miden las percepciones y 25 que miden las expectativas que están divididos en 5 dimensiones: tangibilidad, fiabilidad, capacidad de respuesta, seguridad y empatía. El escalamiento de respuesta es de 7 valores: donde 1 significa que el alumno está en total desacuerdo con la afirmación y 7 si está en total acuerdo. Parasuraman y Zeithaml (1988) reportan una confiabilidad en Alfa de Cronbach de .92 y un análisis de consistencia interna en el que todos los items correlacionaron positivamente con un nivel de significancia de 0.0001 (Parasuraman, 1988). El marco de diseño de muestra se creó considerando como unidad de estudio al estudiante universitario representativo en la población total de la Facultad de Economía de la UANL. Para este fin se procedió a diseñar una muestra irrestricta aleatoria estratificada en dos dimensiones: semestre de carrera $\left(1^{\circ}\right.$, 
$3^{\circ}, 5^{\circ}, 7^{\circ}$ y $9^{\circ}$ semestres) y género (masculino y femenino), dando un total de 10 estratos de clasificación. Así se tomó como base de datos la composición estudiantil de la FAECOUANL para el periodo semestral Agosto-Enero 2020-2021. Se consideró como población objetivo la composición observada en la Tabla 1. La muestra final es de un total de 350 cuestionarios distribuidos entre los estratos como se presenta en la Tabla 2.

Tabla 1. Composición de población objetivo: estudiantes de la carrera de Economía (FAECOUANL).

\begin{tabular}{|c|c|c|c|}
\hline Semestre & Mujeres & Hombres & Total \\
\hline $\mathbf{1}$ & 45 & 103 & 148 \\
\hline $\mathbf{3}$ & 34 & 52 & 86 \\
\hline $\mathbf{5}$ & 28 & 67 & 95 \\
\hline $\mathbf{7}$ & 38 & 47 & 85 \\
\hline $\mathbf{9}$ & 21 & 28 & 49 \\
\hline Total & $\mathbf{1 6 6}$ & $\mathbf{2 9 7}$ & $\mathbf{4 6 3}$ \\
\hline
\end{tabular}

Fuente: Composición estudiantil de semestre Agosto-Enero 20202021 en carrera de Economía (FAECO-UANL) proporcionado por la dirección de la facultad.

Tabla 2. Composición de muestra por diseño irrestricto aleatorio estratificado: alumnos encuestados por semestre y género.

\begin{tabular}{|c|c|c|c|}
\hline Semestre & Mujeres & Hombres & Total \\
\hline $\mathbf{1}$ & 34 & 62 & 96 \\
\hline $\mathbf{3}$ & 29 & 40 & 69 \\
\hline $\mathbf{5}$ & 26 & 47 & 73 \\
\hline $\mathbf{7}$ & 31 & 37 & 68 \\
\hline $\mathbf{9}$ & 19 & 25 & 44 \\
\hline Total & $\mathbf{1 3 9}$ & $\mathbf{2 1 1}$ & $\mathbf{3 5 0}$ \\
\hline
\end{tabular}

Fuente: Elaboración propia usando cuestionario "Estudio de Contexto, Salud, Emocional y Hábitos en Estudiantes de Economía".

Se realizó análisis de brechas de las dimensiones de calidad con el objetivo de mostrar la calidad percibida por parte de los alumnos de la Licenciatura en Economía de la Facultad de Economía de la UANL. Además, se realiza un análisis descriptivo utilizando la media aritmética y la desviación típica estándar con el objetivo de mostrar los valores promedio de las expectativas y percepciones que tienen los estudiantes con respecto a las diferentes dimensiones de calidad.

\section{PRUEBAS DE CONFIABILIDAD}

Como criterio para la confiabilidad del cuestionario SERVQUAL se utilizó el coeficiente de Alfa de Cronbach. Hernández, Fernández y Baptista (2014) sugieren para propósitos de investigación en mediciones sociales los valores aceptables de alfa $\geq 0.7$. El método de consistencia interna basado en el Alfa de Cronbach permite estimar la fiabilidad de un instrumento de medida a través de un conjunto de ítems que se espera que midan el mismo constructo o dimensión teórica. El Alfa de Cronbach de los alumnos de la Facultad de Economía de la UANL fue calculada a partir de las correlaciones de los ítems, es decir, se calculó el Alfa de Cronbach estandarizado donde se toman en cuenta las correlaciones lineales de las preguntas. La Tabla 3 muestra el Alfa de Cronbach para las expectativas y percepciones en conjunto e individualmente para el cuestionario SERVQUAL. Se obtuvo un valor de .9444 para los 50 ítems del cuestionario y valores de .9522 y .9593 para las expectativas y percepciones, lo que significa que el instrumento es sumamente confiable.

Tabla 3. Análisis de fiabilidad (validación estadística) del cuestionario SERVQUAL.

\begin{tabular}{|c|l|}
\hline Elemento (ítems) & Alfa de Cronbach \\
\hline SERVQUAL (50) & .9444 \\
\hline Expectativas (25) & .9522 \\
\hline Percepciones (25) & .9593 \\
\hline
\end{tabular}

Fuente: Elaboración propia usando cuestionario "Estudio de Contexto, Salud Emocional y Hábitos en Estudiantes de Economía”.

En la Tabla 4 se muestran los valores de Alfa de Cronbach dividido por dimensiones que muestran valores en las expectativas entre .7906 y .8869 y para el caso de las percepciones se presentan valores de confiabilidad de entre .7876 y .9148. Las percepciones y las expectativas junto con sus dimensiones presentan buenos valores de Alfa de Cronbach que indican que es confiable el instrumento SERVQUAL para medir la calidad de la Facultad de Economía.

\section{RESULTADOS}

En la Tabla 5 se presentan los datos descriptivos para los ítems y dimensiones del cuestionario SERVQUAL. Las dimensiones en las que los alumnos tienen mayores expectativas son la seguridad y la empatía mientras que las dimensiones con mayores 
percepciones es la tangibilidad y la seguridad. Los ítems que los alumnos presentan altas expectativas son: "Los estudiantes deberían de ser informados sobre la realización de ciertas actividades con tiempo" (item 10), "La calidad del proceso educativo debería ser de alto nivel" (ítem 16) y "El personal docente debería tratar a los estudiantes por igual y con respeto" (item 22). Para el caso de las percepciones son: "El edificio y los alrededores de la Facultad de Economía es moderno y visualmente agradable" (item 2), "Los trabajadores de la Facultad de Economía son profesionales y ordenados" (item 3) y "La calidad del proceso educativo debería ser de alto nivel" (ítem 16).

Tabla 4. Alfa de Cronbach de las dimensiones

\begin{tabular}{|l|c|c|}
\multicolumn{3}{|c|}{ de SERVQUAL. } \\
\hline Dimensión & Expectativas & Percepciones \\
\hline Tangibilidad & .7966 & .7876 \\
\hline Fiabilidad & .8080 & .8401 \\
\hline $\begin{array}{l}\text { Cap. } \\
\text { Respuesta }\end{array}$ & .7906 & .8940 \\
\hline Seguridad & .8745 & .8863 \\
\hline Empatía & .8869 & .9148 \\
\hline $\begin{array}{l}\text { Fuente: Elaboración propia usando cuestionario "Estudio de } \\
\text { Contexto, Salud Emocional y Hábitos en Estudiantes de } \\
\text { Economía". }\end{array}$
\end{tabular}

En la Tabla 6 se presentan la media de cada una de las dimensiones para las expectativas y percepciones. De acuerdo con el cuestionario SERVQUAL para todas las dimensiones se debe de restar el valor dado por los alumnos de las expectativas a las percepciones. En caso de que esta resta sea negativa se dice que tal dimensión es percibida insatisfactoria por los alumnos evaluados. Como se presenta en la tabla las dimensiones con una evaluación más baja son la capacidad de respuesta y la empatía. Esto se debe a que los estudiantes no ven disposición para ayudarlos y no sienten que se les proporciona un servicio rápido por parte de su institución. La tangibilidad es la mejor evaluada por los alumnos que indica que la Facultad de Economía de la UANL cuenta con buenas instalaciones físicas, equipo moderno y buen aspecto de su personal. La calidad global percibida que se calcula a partir del promedio de las diferencias de las percepciones y expectativas de las dimensiones arroja un valor de -.763 , esto indica que la calidad que perciben los estudiantes de la facultad es insatisfactoria. Se usaron pruebas " $t$ " de Satterwhite para medias con diferentes varianzas en muestras emparejadas. Con este criterio se evaluó si las expectativas y percepciones de los estudiantes son significativamente diferentes. La Tabla 7 muestra cada una de las pruebas para cada dimensión: los resultados muestran que para la prueba de dos colas que se utilizó solamente las expectativas y percepciones de la dimensión de tangibilidad no son significativamente diferentes, esto indica que los estudiantes están satisfechos con los elementos tangibles de su facultad. Para las demás dimensiones sí se tienen diferencias significativas con un nivel de significancia del 0.05 .

Este estudio realizado para medir la calidad de servicio percibido a través de SERVQUAL por los estudiantes de la Facultad de Economía de la UANL también se ha aplicado a facultades de ciencias económicas y negocios a través del mundo. Como es el caso de una facultad de Canadá (Chua, 2004) cuyos resultados muestran una calidad de servicio percibido insatisfactorio. Los resultados negativos, como ya se ha mencionado en diferentes universidades, no significa que la universidad brinde un mal servicio ya que el instrumento SERVQUAL mide tanto la percepción de calidad que reciben los clientes (estudiantes) como las expectativas que éstos puedan tener antes de que el servicio sea brindado. El valor negativo de esta variable puede deberse a las altas expectativas que los estudiantes tienen sobre la facultad antes de ingresar a ella. El análisis realizado en la Facultad de Economía de la UANL muestra que la calidad de servicio percibido se encuentra dentro de las facultades mejores evaluadas comparándola con estudios muy similares hechos alrededor del mundo. Aun así sea una universidad muy bien evaluada o no ésta tiene la necesidad de poder observar a través de SERVQUAL cuáles podrían ser sus prioridades y qué puede mejorar para así poder brindar un mejor servicio a sus estudiantes ya que éstos son los 
principales consumidores de los servicios de calidad de las universidades.

Tabla 5. Estadísticas descriptivas de los ítems y dimensiones del instrumento SERVQUAL.

\begin{tabular}{|c|c|c|c|c|c|}
\hline & & \multicolumn{2}{|c|}{ Expectativas } & \multicolumn{2}{|c|}{ Percepciones } \\
\hline Dimensiones e ítems & & Media & DE & Media & $\mathbf{D E}$ \\
\hline \multirow[t]{5}{*}{ Tangibilidad } & & 6.093 & .989 & 6.004 & 0.901 \\
\hline & Ítem 1 & 6.407 & 1.091 & 6.014 & 1.126 \\
\hline & Item 2 & 5.842 & 1.415 & 6.22 & 1.144 \\
\hline & Ítem 3 & 5.536 & 1.612 & 6.089 & 1.071 \\
\hline & Ítem 4 & 6.592 & 0.902 & 5.681 & 1.268 \\
\hline \multirow[t]{7}{*}{ Fiabilidad } & & 6.326 & 0.774 & 5.628 & .986 \\
\hline & Ítem 5 & 6.155 & 1.248 & 5.841 & 1.107 \\
\hline & Ítem 6 & 6.424 & 1.03 & 5.387 & 1.383 \\
\hline & Ítem 7 & 6.480 & 0.943 & 5.644 & 1.308 \\
\hline & Ítem 8 & 5.874 & 1.47 & 5.641 & 1.288 \\
\hline & Ítem 9 & 6.309 & 1.046 & 5.264 & 1.576 \\
\hline & Ítem 10 & 6.727 & 0.805 & 5.954 & 1.244 \\
\hline \multirow[t]{4}{*}{ Capacidad de respuesta } & & 6.411 & .861 & 5.154 & 1.394 \\
\hline & Ítem 11 & 6.542 & 0.939 & 5.089 & 1.528 \\
\hline & Ítem 12 & 6.370 & 1.066 & 5.112 & 1.593 \\
\hline & Ítem 13 & 6.325 & 1.071 & 5.261 & 1.479 \\
\hline \multirow[t]{7}{*}{ Seguridad } & & 6.565 & 0.675 & 5.745 & 1.055 \\
\hline & Ítem 14 & 6.562 & 0.834 & 5.569 & 1.432 \\
\hline & Ítem 15 & 6.605 & 0.801 & 5.629 & 1.336 \\
\hline & Ítem 16 & 6.696 & 0.798 & 6.172 & 1.10 \\
\hline & Ítem 17 & 6.643 & 0.797 & 5.147 & 1.596 \\
\hline & Ítem 18 & 6.490 & 0.955 & 5.974 & 1.283 \\
\hline & Ítem 19 & 6.407 & 0.974 & 5.954 & 1.208 \\
\hline \multirow[t]{7}{*}{ Empatía } & & 6.544 & 0.725 & 5.572 & 1.21 \\
\hline & Ítem 20 & 6.388 & 1.042 & 4.94 & 1.67 \\
\hline & Ítem 21 & 6.503 & .897 & 5.563 & 1.47 \\
\hline & Ítem 22 & 6.744 & .801 & 5.997 & 1.292 \\
\hline & Ítem 23 & 6.353 & 1.032 & 5.712 & 1.312 \\
\hline & Ítem 24 & 6.63 & .811 & 5.234 & 1.658 \\
\hline & Ítem 25 & 6.674 & .836 & 5.997 & 1.235 \\
\hline
\end{tabular}

Fuente: Elaboración propia usando cuestionario "Estudio de Contexto, Salud Emocional y Hábitos en Estudiantes de Economía".

Tabla 6. Análisis de brechas de las dimensiones del cuestionario SERVQUAL.

\begin{tabular}{|l|l|l|l|}
\hline Dimensiones & Expectativas & Percepciones & Brecha \\
\hline Tangibilidad & 6.093 & 6.004 & -.084 \\
\hline Fiabilidad & 6.326 & 5.628 & -.697 \\
\hline Capacidad de Respuesta & 6.411 & 5.154 & -1.241 \\
\hline Seguridad & 6.565 & 5.745 & -.819 \\
\hline Empatía & 6.544 & 5.572 & -.961 \\
\hline Calidad global percibida & & -.763 \\
\hline
\end{tabular}

Fuente: Elaboración propia usando cuestionario "Estudio de Contexto, Salud Emocional y Hábitos en Estudiantes de Economía". 
Tabla 7. Prueba " $\mathrm{t}$ " de Satterwhite muestras emparejadas para las dimensiones de SERVQUAL.

\begin{tabular}{|l|l|l|l|l|}
\hline Dimensiones & Expectativas & Percepciones & Estadístico $\boldsymbol{t}$ & Valor $\boldsymbol{p}$ \\
\hline Tangibilidad & 6.093 & 6.004 & 1.2329 & .2185 \\
\hline Fiabilidad & 6.326 & 5.628 & 10.9244 & .0000 \\
\hline Capacidad de Respuesta & 6.411 & 5.154 & 14.0485 & .0000 \\
\hline Seguridad & 6.565 & 5.745 & 12.9325 & .0000 \\
\hline Empatía & 6.544 & 5.572 & 12.7205 & .0000 \\
\hline
\end{tabular}

Fuente: Elaboración propia usando cuestionario "Estudio de Contexto, Salud Emocional y Hábitos en Estudiantes de Economía".

Al enfocarse en la percepción de calidad de los alumnos con respecto a los servicios brindados se asegura que se mantengan los factores de atracción y retención de talento para poder construir economistas de alta calidad.

A manera de comparación con respecto a los valores de calidad percibida en las escuelas mencionadas en este estudio se encuentra que en una lista de menor a mayor calidad percibida se encuentra: la Universidad King Saud en Arabia Saudita con calidad insatisfactoria de -1.552 (Alhabeeb, 2015), la Universidad de Belgrade en Serbia con -1.212 (Krsmanovic et al., 2014), la Universidad Ryerson en Canadá con -.966 (Chua, 2004), la escuela de negocios de la Universidad de Leed en el Reino Unido con -.846 (Barnes, 2007), la Facultad de Economía de la UANL con un valor de -.763, una facultad de negocios de Estados Unidos con -.66 (Pariseau, 1997) y finalmente la universidad de Pavia en Italia con un valor de -.6 (Wael, 2015).

Algunas de estas universidades comparándolas por dimensiones son similares entre ellas pero es importante saber que cada universidad debería de enfocarse en los aspectos donde fue evaluado más bajo y ver qué mejoras podría aplicar en pro de sus estudiantes para mejorar la calidad global.

\section{CONCLUSIONES}

El presente artículo tiene como objetivo mostrar la calidad percibida de los alumnos de la Facultad de Economía de la UANL, así como determinar los valores de calidad que los alumnos asignan a las diferentes dimensiones de calidad. Se utiliza como metodología una estrategia de muestreo estadístico que permite construir una muestra representativa para los estudiantes considerando 10 estratos diferenciados entre los distintos sexos (hombres y mujeres) y semestres $\left(1^{\circ}, 3^{\circ}, 5^{\circ}, 7^{\circ}\right.$ y $9^{\circ}$ semestre). Para este fin se utilizó la media aritmética y la desviación típica estándar de los ítems, así como las diferencias de las percepciones y expectativas de las dimensiones de calidad de la escala SERVQUAL (Parasuraman, 1988). De acuerdo con los resultados, los ítems que los alumnos presentan en promedio más altas expectativas son: "Los estudiantes deberían de ser informados sobre la realización de ciertas actividades con tiempo" (ítem 10), "La calidad del proceso educativo debería ser de alto nivel" (ítem 16) y "El personal docente debería tratar a los estudiantes por igual y con respeto" (item 22). Para el caso de las percepciones son: "El edificio y los alrededores de la Facultad de Economía es moderno y visualmente agradable" (ítem 2), "Los trabajadores de la Facultad de Economía son profesionales y ordenados" (ítem 3) y "La calidad del proceso educativo debería ser de alto nivel" (item 16). Además, se muestra que las dimensiones en las que los alumnos tienen mayores expectativas son la seguridad y la empatía mientras que las dimensiones con mayores percepciones es la tangibilidad y la seguridad.

Todas las dimensiones a excepción de la tangibilidad tienen diferencias significativas entre las percepciones y expectativas con un nivel de significancia del .05 donde las expectativas son mayores que las percepciones en todos los casos. Se utiliza el Alfa de Cronbach para probar la confiabilidad del instrumento SERVQUAL que da valores por encima del nivel de confiabilidad sugerido. Finalmente la calidad global percibida que se calcula a partir del promedio de las diferencias de las percepciones y expectativas de las dimensiones arroja un valor de -.763 , esto indica que la calidad que perciben los estudiantes de la facultad es insatisfactoria.

\subsection{CONFLICTO DE INTERÉS}

Toda la información procesada en este trabajo fue sujeta a consentimiento firmado por los participantes quienes acordaron de manera 
voluntaria ser parte del estudio. La clasificación de los datos fue en estricto proceso de protección de datos cegando la información confidencial y personal de los alumnos y garantizando la anonimidad de las respuestas obtenidas en cada dimensión. Los autores declaran que el presente trabajo es libre de cualquier otro conflicto de interés derivado de su método, datos y resultados.

\section{AGRADECIMIENTOS}

Los autores agradecen el apoyo de trabajo de la licenciada Brenda G. Medina Soto, psicóloga de la Facultad de Economía, quien colaboró en la selección de instrumentos y aplicación de los cuestionarios. Agradecemos el apoyo del doctor David Hernández Barajas quien proporcionó la financiación de los instrumentos de aplicación de los cuestionarios. Así también los autores agradecen las facilidades concedidas por la Dirección de la Facultad de Economía de la UANL para la realización del presente estudio. Los resultados son únicamente responsabilidad de los autores y no reflejan la postura de ninguna institución vinculante. 


\section{REFERENCIAS}

Aghamolaei, Teamur y Zare, Shahram. (2008). Quality gap of educational services in viewpoints of students in Hormozgan University of medical sciences. BMC medical education. 8. 34. 10.1186/1472-6920-8-34.

Ahmed, I., Nawaz, M., Ahmad, Z., Ahmad, Z., Shaukat, M.Z., Usman, A.K., Wasim-ul-Rehman y Ahmed, N. (2010). Does service quality affect students' performance? Evidence from institutes of higher learning. African Journal of Business Management, 4, 2527-2533.

Barnes, B. R. (2007). Analyzing Service Quality: the case of post-graduate Chinese students. Total Quality Management \& Business Excellence, 18(3), 313-331.

Bautista, M. G., Moreano, E. Z. y Vaca, M. Z. (2017). El Modelo Servqual y su incidencia en el nivel de satisfacción de la carrera de economía de la Universidad Nacional De Chimborazo. European Scientific Journal, 13(25), 339.

Chua C. (2004). Perception of Quality in Higher Education. Proceedings of the Australian Universities Quality Forum 2004. AUQA Occasional Publication.

Chui, T. B., Ahmad, M. S. B., Bassim, F. B. A. y Zaimi, N. B. A. (2016). Evaluation of Service Quality of Private Higher Education Using Service Improvement Matrix. Procedia-Social and Behavioral Sciences, 224, 132-140.

Cuthbert, P. (1996a). Managing service quality in HE: is SERVQUAL the answer? part 1. Managing Service Quality, 6(2), 211-216.

Djonlagic, Sabina y Dedić, Samira. (2015). Quality assessment in higher education using the SERVQUALQ model. Management (Croatia), 20, 39-57.

Foropon, Cyril y Seiple, Ruth y Kerbache, L. (2013). Using Servqual to Examine Service Quality in the Classroom: Analyses of Undergraduate and Executive Education Operations Management Courses. International Journal of Business and Management, 8.

García-Mestanza, Josefa. (2008). "Comparativa entre distintos sistemas de medición de calidad de servicio". EsicMarket, 130, 57-97.

Goran, D. (2014). Analysis of SERVQUAL dimensions: the case of the School of Economics and Business. University of Sarajevo. https://www.academia.edu/31599368/ANALYSIS_OF_SERVQUAL_DIMENSIONS_THE_CA SE_OF_THE_SCHOOL_OF_ECONOMICS_AND_BUSINESS_UNIVERSITY_OF_SARAJE VO.

Guzman, P. J. J. (2018). Calidad en servicios educativos mediante aplicación de Servqual, caso Universidad Técnica de Machala. [Repositorio Digital de la UTMACH]. http://repositorio.utmachala.edu.ec/handle/48000/13236

Hernández, R., Fernández, C. y Baptista, P. (2014). Metodología de la investigación. Editorial McGraw-Hill Interamericana.

Ibrahim, Essam y Wang, Lee y Hassan, Abeer. (2013). Expectations and Perceptions of Overseas Students towards Service Quality of Higher Education Institutions in Scotland. International Business Research, 6.

Jaquez, S. y Godínez, L. (2017). Evaluación de la calidad en el servicio en Centro de Enseñanza de Idiomas del Campus Caborca de Universidad de Sonora mediante SERVQUAL. Invurnus, 12(2), $18-24$.

Kanakana, M. G. (2014). Assessing Service Quality in Higher Education using the SERVQUAL tool. Proceedings of the 2014 International Conference on Industrial Engineering and Operations management, Indonesia.

Krsmanovic, M., Horvat, A. y Ruso, J. (2014). Application of SERVQUAL model in high education. Paper presented at the 11th International conference "Standardization, porotypes and quality: A means of Balkan countries collaboration".

Parasuraman, A., Zeithaml, V. A. y Berry, L. L. (1985). A Conceptual Model of Service Quality and its Implications for Future Research. Journal of Marketing, 49(4), 41-50. 
Parasuraman, Valarie A. y Zeithaml, A. (1988). SERVQUAL: A Multiple-Item Scale for Measuring Consumer Perceptions of Service Quality. Journal of Retailing, 64(1), 12-40

Sahney, S., Banwet, D.K. y Karunes, S. (2004). A SERVQUAL and QFD approach to total quality Education-A student perspective. International Journal of Productivity and Performance Management, 53(2), 143-166.

Pariseau, Susan E. y McDaniel, J.R. (1997). "Assessing service quality in schools of business". International Journal of Quality \& Reliability Management, 14(3), 204-218.

UNICEF. (s.f.). Educación básica e igualdad entre los géneros. https://www.unicef.org/spanish/education/index_quality.html

Wael, T. (2015). Using Servqual Model to Assess Service Quality and Students Satisfaction in Pavia University-Italy. International Journal of Research in Business Studies and Management, 2(3), $24-31$.

Zafiropoulos, C. y Vrana, V. (2008). Service Quality Assessment in a Greek Higher Education Institute. Journal of Business Economics and Management, 9(1), 33-45. 